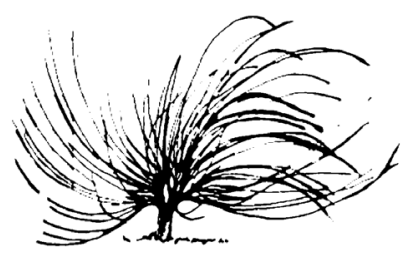

\title{
Avatares del principio de placer y principio de realidad en la educación
}

\author{
Mónica Morales Barrera ${ }^{1}$ \\ Facultad de Estudios Profesionales Aragón \\ México \\ monicamoralesba56@gmail.com
}

\begin{abstract}
Resumen
En su Presentación Autobiográfica (1925), Sigmund Freud advierte que él no escribió nada sobre educación; sin embargo, dice que los descubrimientos que en psicoanálisis se han realizado en el periplo por la exploración del inconsciente han interesado a otras disciplinas, entre las que se encuentra la pedagogía. En los pocos artículos en los que Freud alude a la educación, habla de esta en términos de sueños y esperanzas; en general, la concibe como una suerte de profilaxis de las neurosis y de las perversiones, en tanto el psicoanálisis actuaría a posteriori en el nivel terapéutico. Solo en uno de sus artículos, Formulaciones sobre los dos principios del acaecer psíquico (1911) -principio de displacer-placer y principio de realidad-, Freud asigna a la educación una función específica; en sus propias palabras, dice que esta “... puede describirse, sin más vacilaciones, como la incitación a vencer el principio de placer y a sustituirlo por el principio de realidad" (1989a, p. 228). Esta breve tesis contiene la esencia del poder humanizante de la educación, que hace posible que un sujeto ingrese
\end{abstract}

Recibido: 20 de enero de 2018. Aprobado: 24 de setiembre de 2018.

http://dx.doi.org/10.15359/rep.13-2.3

1. Dra. en Pedagogía por la Universidad Nacional Autónoma de México (UNAM) y Mtra. en Teoría psicoanalítica por el Centro de Investigaciones y Estudios Psicoanalíticos (CIEP). Profesora de tiempo completo en la licenciatura de Pedagogía de la FES Aragón. 
al circuito de la cultura, al poner coto a los impulsos autodestructivos promovidos por el principio del placer. Analizar dicha tesis es el propósito de este escrito.

Palabras claves: psicoanálisis, Freud, educación, principio de placer, principio de realidad.

\begin{abstract}
Sigmund Freud in his Autobiographical Presentation (1925) explicitly warns that he did not write anything about education; however, he says that the discoveries achieved in psychoanalysis in the journey through the exploration of the unconscious have interested other disciplines including pedagogy. In the few articles in which Freud alludes to this topic, he speaks of education in terms of dreams and hopes; he generally conceives education as a kind of prophylaxis of neuroses and perversions; nevertheless, psychoanalysis would act a posteriori at a therapeutic level. Only in one of his article Formulations on the Two Principles of Psychic Accomplishment (1911) -principle of displeasure and pleasure and principle of reality- Freud assigns education a specific function; in his own words: "Education can be described, without hesitation, as the incitement to overcome the pleasure principle and replace it with the principle of reality" (1989a, p. 228). This brief thesis contains the essence of the humanizing power of education that enables the subject to enter the circuit of culture by putting a stop to the self-destructive impulses promoted by the pleasure principle. Analyzing this thesis is the purpose of this paper.
\end{abstract}

Keywords: psychoanalysis, Freud, education, principle of pleasure, principle of reality.

\title{
Freud y la pedagogía
}

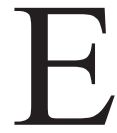

n su Presentación autobiográfica (1925), Sigmund Freud señala que él no colaboró expresamente en la aplicación del psicoanálisis a la pedagogía, pero que "era natural que los descubrimientos analíticos sobre la vida sexual y el desarrollo anímico 
de los niños reclamaran la atención de los educadores y les hicieran ver sus tareas bajo una nueva luz" (1989f, p. 65). De hecho, a los educadores y pedagogos se les demanda saber sobre su objeto de intervención y las formas de acción educativa que pueden ejercer; así, lo descubierto por el psicoanálisis es una fuente imprescindible de conocimiento. De modo paralelo, esta disciplina puede proveer a los agentes educativos de una sensibilidad sobre su propio inconsciente, que les ayude a comprender su propio actuar pedagógico y, a su vez, les brinde una nueva lectura de otros escenarios educativos.

De toda la obra freudiana, solo unos cuantos artículos abordan explícitamente el vínculo entre psicoanálisis y educación, a saber: Formulaciones sobre los dos principios del acaecer psíquico (1911), Introducción a Oskar Pfister (1913); El interés del psicoanálisis (1913); Prólogo a Augusto Aichhorn (1925); Conferencia Núm. 34: Esclarecimientos, aplicaciones y orientaciones (1933).

En Formulaciones sobre los dos principios del acaecer psíquico (1989a), artículo base de este ensayo, Freud propone dos mecanismos que rigen la conducta humana: el principio de placer y el principio de realidad. El primero de ellos lo elabora a partir de lo que advierte en la clínica de pacientes neuróticos, es decir, el sujeto expulsa de su conciencia aspectos que le resultan perturbadores, refugiándose en el principio de placer y apartándose de la realidad. El segundo concepto apunta a la posibilidad que el sujeto tiene a la obtención de satisfacción por vías socialmente aceptadas. En este artículo, Freud hace un recorrido por diferentes ámbitos de la vida humana, observando cómo operan estos dos principios: el desarrollo de los órganos sensoriales y la aparición del pensamiento propiamente dicho; la pulsión sexual, la fantasía, el arte y la ciencia; el deseo inconsciente y el examen de realidad, y, por supuesto, la educación.

Dos años más tarde, en Introducción a Oskar Pfister (1913), Freud expone una idea que quita a los médicos la exclusividad del ejercicio del psicoanálisis y hace una defensa sobre el derecho de los legos a servirse del conocimiento psicoanalítico; afirma que "el ejercicio del psicoanálisis exige mucho menos una instrucción médica que una preparación psicológica y una libre visión humana" (1989b, p. 352). Esta idea se engendra en la cercana amistad y correspondencia con Oskar Pfister, sacerdote protestante y educador en Zurich, quien fue un defensor de las concepciones psicoanalíticas. En ocasión de esta defensa, Freud distingue claramente 
las funciones de cada una de estas disciplinas: la educación sería una suerte de profilaxis que intenta prevenir desenlaces indeseados como la neurosis y las perversiones, en tanto que el psicoanálisis actuaría a posteriori de manera correctiva en una suerte de "poseducación".

En el mismo año se publica El interés por el psicoanálisis, artículo que hace un recorrido por los aportes de la disciplina psicoanalítica en otros ámbitos del saber como la psicología, la religión, la lingüística y, entre ellas, la pedagogía. En el inciso "el interés pedagógico", Freud enumera algunos de los hallazgos respecto del alma infantil, como la disposición perversa polimorfa, y de las contribuciones de estos, por medio de la sublimación y de las formaciones reactivas, a logros culturales importantes. En síntesis, el pedagogo debe allanar el terreno educativo para que dichos procesos sucedan (1989c).

En 1925, casi doce años después de no abordar el tema educativo, Freud escribe Prólogo a August Aichhorn, Verwahrloste Jugend (Juventud descarriada). El libro del educador austriaco Aichhorn trata de un sector de la población bastante problemático. En el prólogo, Freud reconoce la magna labor de los pedagogos frente a la escasa contribución que hace al respecto el psicoanálisis; más bien confía que el paso de los educadores por el diván sería más beneficioso en la intervención con chicos poseedores de esta marca social, que el simple aprendizaje teórico de lo discernido del inconsciente (1989e).

Ocho años más tarde, cuando Freud tenía setenta y siete años escribió una ponencia con tintes de legado: la Conferencia Núm. 34 Esclarecimientos, Aplicaciones y Orientaciones (1933). Entre otras cosas nombra a su hija, Anna Freud, como una de las personas que tomaría la estafeta descuidada por él que fue la educación. Igualmente, expuso una de las preguntas más importantes en ese campo: ¿para qué educamos? Y expresa que una de sus misiones en todo lugar y tiempo es lograr que el niño aprenda "el gobierno de lo pulsional" (1989g, p. 138). Sabemos que no es posible proporcionar al infante toda la libertad de seguir sus impulsos sin limitación alguna, es algo que debe aprender para insertarse en el mundo de la cultura, y esta tarea no puede ser realizada sin interdictos. Prohibir, entre otras funciones, es una de las tareas educativas más difíciles pero estructurantes en la vida psíquica. Mediante su acción, el sujeto conoce la ley, se apropia de ella, lo que le posibilita constituirse como sujeto deseante. En este contexto, la prohibición tiene una valencia positiva: es un acto de amor en lo simbólico, dirigido a 
poner límites al goce angustiante del "todo está permitido", abriendo el camino hacia el placer socialmente regulado. En palabras de Freud, "la educación tiene que buscar su senda entre la Escila de la permisión y la Caribdis de la denegación (frustración)" (1989h, p.138). Aunque Freud escribió poco acerca de la educación, sus hallazgos sobre el inconsciente han incitado a la reflexión entre los profesionales de la pedagogía.

\section{El interés pedagógico por el psicoanálisis}

Durante mi experiencia docente por más de veinte años, en el seminario de Psicoanálisis y Educación, en la licenciatura de Pedagogía en la Facultad de Estudios Superiores Aragón, de la Universidad Nacional Autónoma de México, he discernido cuatro vertientes en las que el psicoanálisis hace sus aportes a la pedagogía.

La primera se refiere a la concepción del hombre dividido por el inconsciente. Tradicionalmente, la idea de hombre ha sido erigida desde ámbitos disciplinarios que ponderan el imperio del exterior como la sociología, la historia, la psicología, la filosofía o la ideología predominante del lugar y de la época de los que se trate. Los descubrimientos del psicoanálisis vinieron a trastocar estos juicios para dar lugar al sujeto dividido por el inconsciente; desde esta perspectiva el hombre pierde su definición como unidad, como individuo (indivisible), como persona (máscara, persona), como prójimo que se define según pautas observables y medibles. El sujeto del inconsciente revela la dependencia significante que mantenemos con un otro inconsciente, presentado en expresiones subjetivas, en fallas en la palabra y localizable en los discursos que pronunciamos.

Respecto al tema educativo, Freud es claro: es necesario conocer aquel con quien se estará trabajando continuamente, es decir, el educando. El método psicoanalítico ha descubierto los deseos, formaciones inconscientes y procesos de la niñez como el complejo de Edipo, el narcisismo, la sexualidad infantil, etc.; conocimientos que sin duda permiten a educadores y pedagogos familiarizarse con ciertas fases del desarrollo infantil y con mociones pulsionales socialmente inservibles o perversas que afloran en el niño. Dice Freud: "Más bien, los agentes educativos deben abstenerse de una sofocación violenta de esas mociones..." (1989b, p. 192) y habría que añadir la promoción de un camino hacia su sublimación, hacia metas socialmente aceptadas. 
La segunda perspectiva es la de la formación profesional. Concebir al sujeto dividido por el inconsciente permite interrogar a los educadores, pedagogos y padres de familia sobre el deseo inconsciente que subyace en su tarea educativa, sea este de enseñar, castigar, escuchar, el deseo de aprender u otro. Así, el esclarecimiento sobre el obrar educativo, donde el pedagogo debe tomar conciencia, primero, mediante el análisis de algunas experiencias educativas utilizando herramientas y conceptos que nos provee la teoría psicoanalítica, y luego, en la medida de lo posible, para el esclarecimiento de la propia práctica educativa.

Quizá la formación de profesionales en la educación sea uno de los campos en los que la incidencia del psicoanálisis sea más trascendente, en términos de prevención de efectos indeseados en el educando. En Introducción a Oskar Pfister, Freud dice: "[El educador] obrará, con ayuda del psicoanálisis, profilácticamente, sobre el niño todavía sano" (1989b, p. 352); es decir, no se trata de una hacer una psicoterapia con el niño, sino de la claridad que tenga el educador sobre su obrar educativo.

La tercera vertiente refiere al entendimiento de los problemas de aprendizaje y al mecanismo de aprendizaje. Por un lado, el psicoanálisis coadyuva al entendimiento de las dificultades de aprendizaje más allá de la orientación cognitiva, tomando en consideración los acaecimientos inconscientes del sujeto involucrado. Por otra parte, el aprendizaje como evento inherente al ser humano, y condicionado multifactorialmente, es un acto que está vinculado con formaciones inconscientes que influyen tanto en el deseo de saber como de no saber.

La cuarta vertiente es su producción teórica. Existen conceptos generados en el seno de la teoría psicoanalítica, que son susceptibles de ser extrapolados a otros campos de conocimiento. Por ejemplo, hay términos clásicos como el de la transferencia y la sublimación, que sirven para desentrañar aspectos como la relación maestro-alumno y los intereses vocacionales, respectivamente. Otros conceptos menos estudiados, como el de la transmisión, dan cuenta de comportamientos culturales e individuales a lo largo del tiempo. Precisamente es en este contexto en el cual inscribimos el título de este ensayo. 


\section{Principio de placer-displacer y principio de realidad}

En Formulaciones sobre los dos principios del acaecer psíquico (1911), Freud dilucida sobre dos mecanismos imbricados en la naturaleza humana que rigen el comportamiento del hombre de la cultura, estos son: el principio de placer-displacer y el principio de realidad. Este esclarecimiento no fue producto de un discernimiento filosófico-reflexivo, sino de sus propias observaciones durante la clínica psicoanalítica, por lo que es necesario hacer un par de advertencias antes de comenzar el análisis de estas formulaciones.

En primer lugar, nos encontramos con la natural apreciación que frecuentemente se realiza sobre palabras de uso común, como placer y realidad, y que es necesario trascender para acceder a estados de conocimiento válidos y rigurosos acordes con las diferentes epistemologías en las que se han edificado. En segundo lugar, es necesario distinguir la concepción freudiana de placer-displacer de otras como la proveniente del filósofo Epicuro, quien identifica el placer con el bien y lo delimita a partir de la ausencia del dolor; esta idea estaría más cerca al principio de realidad que Freud propone. En una carta que este filósofo escribe a Meneceo, cualifica esta noción como algo que debe ser mesurado, por lo que la integra a su concepción de ética. Epicuro dice:

Así, cuando decimos que el placer es fin, no hablamos de los placeres de los corruptos y de los que se encuentran en el goce, como piensan algunos que no nos conocen y no piensan igual, o nos interpretan mal, sino de no sufrir en el cuerpo ni ser perturbados en el alma (Carta a Meneceo).

Desde el punto de vista metapsicológico, el principio de placer-displacer se refiere a todos aquellos procesos anímicos primarios que se gestaron en nuestro psiquismo desde el umbral de nuestra existencia. Aunque tiende a evitar el displacer, este principio solo tiene como objetivo la ganancia de placer. Por ejemplo, el sueño, guardián del dormir, el cual intenta esquivar las impresiones penosas que provocarían en determinado momento el despertar; asimismo, la vida intrauterina, la satisfacción de la pulsión sexual, el fantasear, el juego, el relajo serían otros aspectos gobernados por el principio del placer-displacer. 
El principio de placer-displacer se dispone como una categoría esencialmente económica, en tanto procura el ahorro de energía psíquica; esta idea se relaciona con un proceso de equilibrio entrópico donde la energía se dispersa, por lo que no hay producción alguna; esta es una de las razones por las que Freud vinculó el principio de placer-displacer con la pulsión de muerte, en 1920. Desde esa perspectiva, esta actividad anímica resulta peligrosa para la autoafirmación del organismo frente a las reales dificultades del mundo exterior en el que se requiere un gasto de energía para poder sobrevivir; por ello, dice Freud, hubo que instaurar un nuevo principio que, sin destronar el principio de placer-displacer, guardara relación con el imperio exterior, este es el principio de realidad (1989a, p. 224).

Así, en Más allá del principio de placer (1920), Freud da un giro a esta concepción de placer-displacer, vinculándola con la pulsión de muerte y proponiendo con este "más allá" un enigma, que surgió, como se refiere antes, de la clínica de las neurosis y, curiosamente, de la observación del juego del nietecito de Freud, de dieciocho meses.

En la clínica de las neurosis, Freud encontró durante la cura de sus pacientes algo inexplicable para el sentido común, pues persistían en el sufrimiento vía el síntoma neurótico, en la compulsión a la repetición, en la reacción terapéutica negativa, en las tendencias masoquistas del yo; con lo que comenzó a sospechar que había algún tipo de placer, pero emparentado con la pulsión de muerte, con la búsqueda del apaciguamiento, fin de todas las tensiones (Freud, 1989e).

Por otra parte, al juego del nieto de Freud - cuyo contexto es que su madre está ausente y él lo está cuidando-, se le conoce con el nombre de fort-da, dos sílabas que acompañan el comportamiento del infante que hace arrojar un carretel y lo vuelve a aparecer, dos movimientos que simbolizan el desagrado por la ausencia de la madre y el placer de hacerla regresar. Se trata del lazo de oposición entre la repetición de la pérdida y la aparición del objeto de deseo: dolor y placer. Precisamente, en esta repetición de la vivencia displacentera es cuando el placer se plantea como un enigma, en un más allá de la satisfacción simbólica. El juego del fort-da, además de mostrar un momento constitutivo en la historia del sujeto, descubre que la naturaleza del placer es de otra índole que la del hedonista.

Aunque este juego es paradigmático en la obra de Freud, existen una infinidad de ejemplos en los cuales se observa la diada dolor-placer: 
un niño vive pasivamente los efectos desagradables de una vacuna, pero cuando llega a casa jugará a ser el doctor y tratará de inyectar a quien se deje. También es común encontrar juegos en los que los infantes juegan a ser maestros y regañan a diestra y siniestra a sus víctimas-alumnos. Aquí se distingue el placer de manera activa y se obtiene en el acto de la venganza por la amonestación sufrida pasivamente.

Desde la lectura de Lev Vygotsky (2008), hay muy pocas cosas que pertenecen al juego en sí. El autor explica que el infante reproduce en tal actividad lo que le acontece en la realidad, ya que dicha actividad aporta solo el escenario donde se desplazan los episodios de la vida diaria; Vygotsky afirma que "el juego está más cerca de la recopilación de algo que ha ocurrido realmente, que de la imaginación. Es más bien memoria en acción que una situación nueva e imaginaria" (2008, p. 156). Desde esta perspectiva, el juego es rememoración, es un campo de proyección donde el sujeto tiene la posibilidad de resignificar aquello que su yo desconoce.

Freud discurre que la pulsión tiene una naturaleza conservadora, pues el organismo vivo tiende a reconstruir un estado anterior, dice: "una pulsión sería entonces un esfuerzo, inherente a lo orgánico vivo, de reproducción de un estado anterior que lo vivo debió resignar bajo el influjo de fuerzas perturbadoras externas" (1989d, p. 36); él lo resume en una frase contundente: "La meta de la vida es la muerte" (p. 38), regresar a lo inanimado. En síntesis, en la teoría analítica, la pulsión de muerte se encarga de representar lo perteneciente al inconsciente y que es, por lo tanto, de un registro radicalmente ajeno a toda función vital. Así, la pulsión no necesariamente tiende hacia adelante, hacia la creación, como sucede en el principio de realidad.

La lectura de Jacques Lacan sobre el principio de placer es esclarecedora, corrige este principio, lo transmuta por el concepto de goce y lo distingue del placer, como constituyendo un más allá de él; dice que el goce apunta al exceso, desborda cualquier equilibrio; "la dosis hace al veneno", dice Paracelso; por ello se reduce a no ser más que una instancia negativa que aparece fuera del orden significante, y se exterioriza en la terquedad, en las adicciones, en las compulsiones. Es una suerte de entropía que indica la parte no utilizable de la energía contenida en un sistema, que no puede usarse para producir trabajo. Dice Lacan, "el goce es lo que no sirve para nada" (1985, p. 11). Se trata de la idea de un placer en exceso, sin límites, desmesurado que produce estragos, que conduce a la muerte. 
Dos ejemplos de la literatura ilustrarán este goce sin límites: uno proveniente de un ensayo de Jorge Portilla, Fenomenología del relajo (1956), y el otro de un relato corto denominado Bartleby, el escribiente (publicado en 1853), de Herman Melville, autor también de Moby Dick.

Portilla aborda en su ensayo un aspecto de la moralidad mexicana que ambiciona traerla a plena conciencia: el relajo, el cual ha impactado de manera profunda en la psicología del mexicano. Portilla afirma que este comportamiento tiene el sentido de suspender la seriedad que se define como "el compromiso íntimo y profundo que pacto conmigo mismo para sostener un valor en la existencia" (1997, p. 19); el relajo cancela la respuesta normal al valor y la desliga del compromiso de su realización. En términos generales, se desvaloriza la ley reguladora y trae como corolario la autodestrucción, en palabras del autor, "el relajo, como desvío de valores, bien pudiera ser fórmula de autoaniquilamiento [...] El relajo, conducta de disidencia, puede ser la expresión de una voluntad de autodestrucción" (1984, p. 34).

Asimismo, un digno representante del principio de placer (goce) es el personaje de Bartleby. La trama mínima trascurre en una de las oficinas Wall-Street, a mediados del siglo XIX, el narrador es el jefe de Bartleby, quien está sumamente sorprendido por la respuesta que él invariablemente le da; ante cualquier petición de su jefe, el protagonista responde: "Preferiría no hacerlo" (I would prefer not to). En la realidad efectiva, esta respuesta sería inusual, pero en la realidad psíquica de Bartleby, contiene no poco misterio. El carácter de la contestación es paradójico, por un lado, expresa "el deseo de no hacer algo"; por otro, carece de cualquier impulso vital. De hecho, el resto de la narración continúa igual hasta su próxima muerte al final de la historia. Ahora bien, ¿por qué circunscribir a Bartleby en el principio de placer? Él no muestra ninguna emoción o displacer, no se queja, no tiene demandas, no tiene pasado ni futuro, es como un muerto viviente, a Bartleby le hace falta la falta.

¿Y qué ofrece el principio de realidad? En este rige la función vital con fines adaptativos, de sobrevivencia y de creación. Es un proceso secundario, en el que intervienen la memoria, el lenguaje, el pensamiento, que son herramientas que nos contactan con la realidad, producto de un largo proceso simbólico cultural; lo más importante que 
se gesta en el principio de realidad es la sublimación ${ }^{2}$ del placer, es decir, se transforma la energía libre, entrópica, en energía ligada, dirigida a objetivos específicos. Como dijo el físico Maxwell: para combatir la entropía es necesario el trabajo; Freud diría que en un análisis lo que se puede esperar es un cambio en la vía de satisfacción que va del principio de placer hacia el de realidad; este último está ligado al apremio a la vida derivado de la renuncia que hacen los individuos de los sentimientos eróticos familiares (Freud, 1989, p. 168).

Kilos mortales es un reality show estadounidense donde se observa nítidamente el tortuoso camino de "vencer" el principio de placer y sustituirlo por el principio de realidad. El programa televisivo documenta, desde 2004, el proceso de personas con obesidad mórbida que intentan reducir de peso, mediante una cirugía de bypass gástrico o una manga gástrica, con la asistencia del Dr. Nowzaradan, en Houston, Texas. La característica de estas personas es que, por diversas razones emocionales y traumáticas, están atrapadas en una adicción compulsiva a la comida que evidencia estentóreamente el placer fácil, anulando el principio de realidad que requiere esfuerzo. No obstante, el periplo que ellas han emprendido hacia metas socialmente aceptadas de salud les reporta un placer sublimado al producirse una satisfacción de logro.

\section{Avatares del principio de placer-displacer y principio de realidad en la educación}

En el ámbito de la educación, Freud define en veinticuatro palabras su tesis fundamental respecto a estos dos principios: "La educación puede describirse, sin más vacilaciones, como la incitación a vencer el principio de placer y a sustituirlo por el principio de realidad" (1989a, p. 228). De acuerdo con esto, es una función universal con la cual el principio de placer debe ser reprimido, mediante la diada castigo-recompensa. Freud advierte que estas medidas fracasan cuando el niño mimado sabe que posee o cree poseer de todas maneras el amor de los padres y que no puede perderlo bajo ninguna circunstancia. Como se sabe, el peligro del consentimiento y la sobreprotección tienen la

2 "A esa facultad de permutar la meta sexual originaria por otra ya no sexual, pero psíquicamente emparentada con ella, se le llama la facultad para la sublimación”. Freud (1908). La moral sexual cultural y la nerviosidad moderna. p. 168. 
indeseable consecuencia de favorecer las perversiones, las cuales no encuentran su límite en un acto de amor.

Ciertamente, en el principio de realidad no desaparece el placer, sino que se transmuta y adquiere nuevas cualidades; ya no es una ganancia de placer inmediata y objetiva, sino que se hace un esfuerzo, se posterga la satisfacción por una ganancia de placer a largo plazo y subjetiva; se trata de algo regulado por la cultura que apunta hacia la vida y el trabajo. En palabras llanas: el principio de realidad nace de las exigencias que impone el mundo real cultural, no está del lado del merecimiento, hay que hacer algo para ganarse la vida, lo que constituye un paso grávido de consecuencias, tanto en el desarrollo individual como en la producción de cultura. Esta actividad psíquica, ontogenética, implica la capacidad del organismo de hacerse cargo, ya no solo de lo placentero, sino también de lo verdadero, aunque sea displacentero. Aquí, la ausencia de la satisfacción esperada y la falta subjetiva ocupan un primerísimo lugar en la producción de este principio. Desde el punto de vista económico, el principio de realidad corresponde a una transformación de energía libre, a una ligada, como en el caso del relajo, es decir, la energía psíquica se dirige hacia los objetos, bienes, fines y a otros.

Ahora bien, advertimos que la instauración del principio de realidad no ocurre de forma automática, sino que la educación participa en gran medida. Esta tarea se realiza generalmente por padres y educadores y su misión es preparar a los infantes para afrontar las exigencias del mundo exterior. Es una labor difícil en extremo, ya que supone que los niños logren resignificar las fuentes de placer inmediato, y requiere una gran claridad respecto a la misión educativa, por parte de los padres y educadores, para que no se convierta en un acto de brutalidad.

En todo lugar y tiempo, una de las tareas de la educación es lograr que el niño aprenda el gobierno de lo pulsional (Freud, 1989h, p. 138). Sabemos que no es posible dar al infante toda la libertad de seguir sus impulsos, sin limitación alguna; es algo que debe aprender para insertarse en el mundo de la cultura, y esta tarea no se puede realizar sin interdicciones. Entre otras funciones, prohibir es una de las labores educativas más difíciles, pero estructurantes en la vida psíquica. Mediante su acción, el sujeto conoce la ley, se apropia de ella, lo que le posibilita constituirse como sujeto deseante. En este contexto, la prohibición tiene una valencia positiva: es un acto de amor en lo simbólico, dirigido a 
poner límites al goce angustiante del todo está permitido, abriendo el camino hacia el placer socialmente regulado.

En el terreno psíquico, se educa para que el sujeto no se autodestruya, para que no sea víctima del goce pulsional sin ley que le habita en el principio de la vida. Anny Cordié (1998, p. 318) afirma que conforme el sujeto se civiliza, el "destino pulsional" se desdibuja, y la educación desempeña un papel muy importante en esto. El individuo va encontrando los caminos de la sublimación y de la formación reactiva que dan lugar al carácter, a la moralidad y a la expresión del deseo en la cultura; es decir, aquel debe aprender el gobierno de lo pulsional, aceptar que no todo está permitido, que hay un sistema de prohibiciones que regulan la vida humana y que además tiene un corolario positivo, pues origina el deseo, la falta, motor de la vida.

En el contexto antes descrito, se puede entender por qué Freud no ve con buenos ojos este principio de placer, ligado a este goce mortífero, al que habría que vencer si se quiere que advenga el hombre de la cultura. Por la historia de la humanidad, sabemos que esta subyugación nunca será total, en tanto el principio de placer es un proceso primario y el de realidad un proceso secundario; así, vencer es una tarea titánica o imposible, pues, además, no todos son capaces de sublimar el placer a partir de sensaciones inmediatas. En La moral sexual cultural y la nerviosidad moderna, Freud hizo un examen del antagonismo entre cultura y vida pulsional, en términos universales dice que "nuestra cultura se edifica sobre la sofocación de pulsiones" (1989, pp. 167-168), por lo que la sublimación tiene altos costos y no todos están dispuestos a pagarlo.

Hoy más que nunca el imperio del principio del placer se encuentra vigorizado por la sociedad de consumo que estimula los sentidos fantásticamente. El Internet - maravillosa herramienta de la tecnología digital - es una fuente poderosa de distracciones, ante el cual es difícil procrastinar; esta postergación es una tendencia a devaluar el futuro y sobrevaluar el presente inmediato de placer, es una manera de ponderar la realidad virtual y esconderse del mundo real.

Pero también es posible encontrar el principio de placer acuñado a múltiples actitudes cotidianas en las cuales se observa este goce improductivo, esa energía que no sirve para nada, dice Lacan (1985 p. 11), por ejemplo, en el fenómeno del bullying, en el que se goza con la destrucción subjetiva de un semejante más débil. Se encuentra en 
expresiones como "se me hizo fácil", etc. Todas estas actitudes implican una ganancia de placer con el mínimo esfuerzo, sin fines culturales.

Otra muestra es la procrastinación, este hábito tan cotidiano que nos arrebata y nos conduce a postergar diligencias que deben atenderse, sustituyéndolas por otro tipo de actividades más agradables o irrelevantes. Lo típico es "dejar todo para el último momento", escuchar la famosa frase de niños y adolescentes "ahorita voy", para posponer frecuentemente tareas que no se desean realizar, o estudiar materias que para estudiantes regulares no tienen la menor importancia. Igualmente, en las quejas puede haber una señal de procrastinación, pues se encuentra el pretexto perfecto para no efectuar determinada tarea, hay un gozo pasivo, una energía desperdiciada que no produce trabajo; sin embargo, la queja puede pertenecer al principio de realidad cuando se asume subjetivamente y promueve la acción.

Adquiere especial importancia el principio de placer en el proceso de aprendizaje, pues, con las urgencias escolares de los estudiantes, estos tienden a ser espectadores del conocimiento para aprobar las asignaturas, y pueden convertirse en problemas de aprendizaje. En realidad, el proceso de aprendizaje pertenece al principio de realidad, en tanto el estudiante, ignorante desde la perspectiva socrática, toma un papel activo y disciplinado frente al conocimiento: en primer lugar, le interesa el tema, se hace preguntas, soporta la incertidumbre, no se conforma con una respuesta rápida, se aleja de los lugares comunes, asume que no todo lo entiende y que debe hacer búsquedas intertextuales; cuando comprende los conceptos, tiende a aplicarlos a diferentes situaciones, equivocándose, rectificando, y sobre todo, encuentra placer, aunque también angustia, en todo este proceso.

Desde esta perspectiva, los procesos creativos también pertenecen al principio de realidad, los cuales van más allá de un simple divertimento y de cualquier gratificación inmediata, aquí la imaginación está al servicio de la producción creativa y no está dada en el vacío; Vygotsky lo dice de la siguiente manera: "Crear es fuente de júbilo para el hombre, pero acarrea también sufrimientos conocidos con el nombre de los tormentos de la creación" (2015, p. 47). Más que todo, este sentimiento aparece cuando la idea, la palabra, el sonido se deniega a plasmarse en obra.

En el principio de realidad, el placer se transforma, se trata de un placer sublimado, ya no ligado a las necesidades inmediatas, se difiere 
en el tiempo y está en espera de una sanción por parte del otro social. A este principio corresponde la educación, el arte, la ciencia, la religión y, en general, cualquier producto cultural. Crear es un acto complejo que no surge de manera repentina, sino lenta y paulatina, bajo condiciones de repetición y esfuerzo.

Los espacios educativos son tan solo los escenarios donde puede o no emerger la creatividad, pues penden del capital sociocultural, emocional de cada persona y de talentos cultivados. El acto creador requiere enormes reservas de experiencia acumulada, dice Vygotsky, que son transformadas a partir de la imaginación; por esta razón, menciona este autor, que "la imaginación del niño es más pobre que la del adulto, por su menor experiencia", por lo que llega a la conclusión pedagógica sobre la necesidad de ampliar la experiencia del niño (2015). Pero no cantemos victoria, pues vencer el principio del placer va contra natura, en tanto es un proceso primario, casa del inconsciente y parte esencial de lo que nos hace humanos.

\section{Referencias}

Cordié, A. (1998). El malestar en el docente. Argentina: Nueva Visión. Epicuro. (s. f.). Carta a Meneceo. Recuperado de http://onomazein.letras.uc.cl/Articulos/4/23_Oyarzun.pdf

Freud, S. (1989). La moral sexual "cultural" y la nerviosidad moderna. En Obras Completas (vol. 9, pp. 159-181). Buenos Aires: Amorrortu. (Trabajo original publicado en 1908).

. (1989a). Formulaciones sobre los dos principios del acaecer psíquico. En Obras Completas (vol. 12, pp. 217- 231). Buenos Aires: Amorrortu. (Trabajo original publicado en 1911).

. (1989b). Introducción a Oskar Pfister. En Obras Completas (vol. 12, pp. 347- 353). Buenos Aires: Amorrortu. (Trabajo original publicado en 1913).

. (1989c). El interés por el psicoanálisis. En Obras Completas (vol. 13, pp.165-192). Buenos Aires: Amorrortu. (Trabajo original publicado en 1913).

. (1989d). Más allá del principio del placer. En Obras Completas (vol. 18, pp. 1-62). Buenos Aires: Amorrortu. (Trabajo original publicado en 1920). 
. (1989e). El problema económico del masoquismo. En Obras Completas (vol. 19, pp. 161-176). Buenos Aires: Amorrortu. (Trabajo original publicado en 1924). . (1989f). Prólogo a Augusto Aichhorn. En Obras Completas (vol. 19, pp. 296-297). Buenos Aires: Amorrortu. (Trabajo original publicado en 1925). . (1989g). Presentación autobiográfica. En Obras Completas (vol. 20, pp. 1-66). Buenos Aires: Amorrortu. (Trabajo original publicado en 1925).

. (1989h). Conferencia Un. 34: Esclarecimientos, aplicaciones y orientaciones. En Obras Completas (vol. 22, pp. 126-145). Buenos Aires: Amorrortu. (Trabajo original publicado en 1933).

Lacan, J. (1985). El Seminario de Jacques Lacan libro 20 Aún. Barcelona-Buenos Aires-México: Paidós.

Melville, H. (1998). Bartleby, el escribiente. (Trad. J. L. Borges). Madrid: Alianza.

Portilla, J. (1997). Fenomenología del relajo. México: Fondo de Cultura Económica.

Vygotsky, L. (2008). El desarrollo de los procesos psicológicos superiores. Barcelona: Biblioteca de Bolsillo. . (2015). La imaginación y el arte en la infancia. México: Ediciones Coyoacán. 